\title{
OPEN Quantitative parameter mapping of contrast agent concentration and relaxivity and brain tumor extracellular $\mathrm{pH}$
}

Yuki Matsumoto ${ }^{1 \bowtie}$, Masafumi Harada ${ }^{1}$, Yuki Kanazawa ${ }^{1}$, Yo Taniguchi $^{2}$, Masaharu Ono ${ }^{2} \&$ Yoshitaka Bito ${ }^{2}$

In clinical magnetic resonance imaging, gadolinium-based contrast agents are commonly used for detecting brain tumors and evaluating the extent of malignancy. We present a new method to evaluate relaxivity $(r 1)$ and contrast agent concentration separately in contrast-enhanced lesions using quantitative parameter mapping (QPM). Furthermore, we also aimed to estimate the extracellular $\mathrm{pH}(\mathrm{pHe})$ of tumor lesions. We demonstrated that it is possible to evaluate pathophysiological tumor changes due to therapeutic efficacy by measuring $\mathrm{r} 1$ in contrast-enhanced lesions. In this study, the primary brain tumor group showed significantly higher $r 1$ values than other brain disease groups $(P<0.001)$. Moreover, mean $\mathrm{pHe}$ value showed a trend for tumor malignancy having a lower $\mathrm{pHe}$ value and primary brain tumor having a significantly lower $p H e$ than other brain diseases $(P<0.001)$. Our results might suggest that QPM can separately quantify $r 1$ and $C A$ concentration in brain tumors and that pHe brain tumor mapping could serve as a tumor biomarker. In conclusion, our method has potential clinical applications for assessing the treatment effects.

The effect of contrast enhancement by a gadolinium (Gd)-based contrast agent (CA) depends on both its concentration and longitudinal relaxivity (r1) in lesions. It is well known that the r1 of a Gd-based CA is affected by the rate of exchange interaction of water protons, which is influenced by factors around the lesion area, such as $\mathrm{pH}$, temperature, and diffusivity ${ }^{1-3}$. Therefore, the $\mathrm{r} 1$ of a CA may reflect the biological environment of the lesion and act as an index of the lesion microenvironment. The pharmacokinetic parameter of Gd-based CAs is spin-lattice relaxation time constant $\left(T_{1}\right)$-shortening because of leakage to extracellular tissues from cerebral vessels owing to blood-brain barrier destruction, which allows the CA to leak out into the extracellular space ${ }^{4}$. The mechanism of the shortened-T1 is mainly due to dipole-dipole interactions of coordinated water molecule with the $\mathrm{Gd} 3+$ complex $^{5,6}$. Furthermore, the acidic environment caused by the anabolic metabolism in the extracellular space can act as a physical source for $\mathrm{Gd} 3+$ relaxation changes, as the hydration state $(\mathrm{q})$ of molecular CAs plays a role in modulating relaxation changes under acidic conditions.

The concentration of CA and CA's $\mathrm{r} 1$ should be independently measured because CA concentration in a lesion depends on several factors including pathophysiological conditions. Quantitative parameter mapping $(\mathrm{QPM})$ is a recently proposed method among synthetic magnetic resonance imaging (MRI) techniques ${ }^{7}$; it uses three-dimensional (3D) RF-spoiled gradient-echo pulse sequences with multiple repetition times, echo times, and flip angle values. The intensity function of the rapid imaging of RF-spoiled gradient echo is then formulated using computer simulation based on Bloch equations. This allows simultaneously acquisition of multiple MR parameter maps, such as T1 and T2 maps, by applying a pulse sequence in which the intensity depends on these MR parameters. One of the important advantages of QPM is that both relaxation and quantitative susceptibility mapping (QSM) can be independently obtained. We considered that QPM before and after CA injection would allow separate quantification of both $\mathrm{r} 1$ and CA concentration using two parameters derived from subtracted longitudinal relaxation rate (R1) and QSMs.

Several studies have shown $\mathrm{pH}$ dependency of relaxivity measurements using $\mathrm{pH}$-responsive $\mathrm{CAs}^{8-11}$. Although it is possible to assess $\mathrm{pH}$ changes by measuring relaxation rates, few studies have demonstrated the relationship between relaxation rate and $\mathrm{pH}$ of commercially available Gd-based $\mathrm{CAs}^{8,9}$. 


\begin{tabular}{|l|l|l|l|}
\hline Age (years) & Sex & Clinical diagnosis & Group \\
\hline 79 & F & Radiation necrosis \#1 & Radiation necrosis \\
\hline 61 & F & Radiation necrosis \#2 & Radiation necrosis \\
\hline 72 & F & Radiation necrosis \#3 & Radiation necrosis \\
\hline 68 & M & Metastasis \#1 & Metastasis \\
\hline 72 & F & Metastasis \#2 & Metastasis \\
\hline 74 & M & Metastasis \#3 & Metastasis \\
\hline 47 & F & Metastasis \#4 & Metastasis \\
\hline 49 & F & Anaplastic astrocytoma & Primary brain tumor \\
\hline 76 & F & Glioblastoma & Primary brain tumor \\
\hline
\end{tabular}

Table 1. Patient data.

This study aimed to separately assess the effect of CA's $r 1$ value and concentration to evaluate the possibility of a new index for $\mathrm{r} 1$ and measure extracellular $\mathrm{pH}$ ( $\mathrm{pHe}$ ) values to obtain pathophysiological information on brain tumors. Therefore, we performed a phantom experiment to investigate the $\mathrm{r} 1$ value of a commercially available Gd-based CA, Gd-BTDO3A, and evaluated the relationship between $\mathrm{r} 1$ and $\mathrm{pH}$ to develop a $\mathrm{pH}$ calibration curve at each $\mathrm{r} 1$ value in Gd-BTDO3A using a non-linear function, as previously reported ${ }^{9}$.

\section{Methods}

Subjects and data acquisition. All clinical investigations were conducted according to the principles expressed in the Declaration of Helsinki. To verify that the pHe map can be measured both before and after CA injection, patients with brain tumor (radiation necrosis: three lesions in three patients, brain metastasis: twelve lesions in four patients, primary brain tumor: two lesions in two patients), all of whom provided informed consent, underwent MRI (Table 1). This study was approved by our institutional review board (Tokushima University Hospital), and all MRI data acquired using a 3-T system (FUJIFILM Healthcare Corporation, Japan) with a 32-element-phased array receive coil. QPM employs 3D partially radio frequency (RF)-spoiled steady state gradient-echo (3D-pRSSG) methods with multiple repetition time, echo-time, and flip angle values. To achieve adequate $\mathrm{T} 1$ and $\mathrm{T} 2$ relaxation times, imaging parameters are optimized using the law of error propagation with target relaxation times at $3 \mathrm{~T}$. In addition, the first RF excitations (up to 50 cycles) were skipped to reach steady state. Imaging parameters were as follows: echo times, $4.5-36.8 \mathrm{~ms}$; repetition times, $10-41.3 \mathrm{~ms}$; and flip angles, $10^{\circ}-40^{\circ}$. Image resolution was $0.94 \times 0.94 \times 2 \mathrm{~mm}$ with a $240 \times 240 \mathrm{~mm}^{2} \mathrm{FOV}$. Parallel imaging was used to reduce overall scan time, and the acceleration factor was $1.9 \times 1.9(\mathrm{RL} \times \mathrm{AP})$. CA amounted to $0.1 \mathrm{mmol} / \mathrm{kg}$ of body weight and was injected into the patient's vascular system. Whole-brain acquisition was then performed using the same imaging parameters as pre-injection. Note that the whole brain was scanned $\geq 1 \mathrm{~min}$ after CA injection. Total scan time was under $19 \mathrm{~min}$, both before and after CA injection. In addition, a susceptibility map was used, which was reconstructed using the QSM algorithm with multi-echo images, as part of the QPM dataset. Multi echoes were used (five echoes, $4.5-36.8 \mathrm{~ms}, \Delta \mathrm{TE}=6.9 \mathrm{~ms}$ ), with repetition time and flip angle of $40 \mathrm{~ms}$ and $10^{\circ}$; other imaging parameters were the same as with the QPM dataset.

Phantom experiment for $\mathrm{pH}$ calibration. $\mathrm{r} 1$ is generally defined as the slope of the resulting fit from a linear regression of the measured R1 (i.e., 1/T1) of the tissue and CA concentration ${ }^{12,13}$ as follows:

$$
\begin{gathered}
R 1_{\text {post }}=R 1_{\text {pre }}+r 1 \times C A \\
\therefore r 1=\frac{R 1_{\text {post }}-R 1_{\text {pre }}}{C A}
\end{gathered}
$$

where $\mathrm{R} 1_{\text {pre }}$ and $\mathrm{R} 1_{\text {post }}$ denote the $\mathrm{R} 1$ values of the tissue before and after $\mathrm{CA}$ injection, respectively. CA denotes the CA concentration. The relaxivity $\mathrm{r} 1$ can thus be estimated by measuring $\mathrm{R} 1_{\text {pre }}, \mathrm{R} 1_{\text {post }}$, and $\mathrm{CA}$, as shown in Eq. (1b). Here, the measured relaxivity value is different in acidic $\mathrm{pH}$; thus, the empirical relationship between those values must be investigated to calculate the $\mathrm{pHe}$. To validate $\mathrm{r} 1$ depending on the $\mathrm{pHe}$, a phantom experiment was then performed using a $\mathrm{pH}$ buffer solution, and $\mathrm{r} 1$ at each $\mathrm{pH}$ acidity was measured. We prepared various $\mathrm{pH}$ solution samples of known acidity ( $\mathrm{pH}$ range of 6.0-7.8), including the CA Gd-BTDO3A (Gadovist, Bayer HealthCare) at different concentrations $(0.1,0.2,0.4$ and $0.5 \mathrm{mmol})$. The $\mathrm{pH}$ of these samples was achieved by mixing sodium dihydrogen phosphate dihydrate $(0.2 \mathrm{mmol})$ and di-sodium hydrogen phosphate 7-hydrate $(0.2 \mathrm{mmol})$ solutions. Additionally, real $\mathrm{pH}$ solutions were measured with a calibrated HI $2020-01 \mathrm{pH}$ meter (HANNA instruments, USA). Then, the sample containers were placed in the MR exam table so that the axes of the sample containers were perpendicular to the main field, and a 3 T MRI system (FUJIFILM Healthcare Corporation, Japan) used at room temperature $\left(20^{\circ} \mathrm{C}\right)$ and body temperature $\left(37^{\circ} \mathrm{C}\right)$. To measure the T1, a single slice of a coronal scan was obtained using a fast spin-echo based inversion recovery (FSE-IR) with the following parameters. Inversion time (TI) was performed at 40, 100, 160, 240, 300, 400, 500, 600, 800, 1000, 1200, 1500, and $2000 \mathrm{~ms}$. Then, $7000 \mathrm{~ms}$ of TR was chosen to assure TR $\gg \mathrm{T} 1$. The shortest possible effective echo-time value 


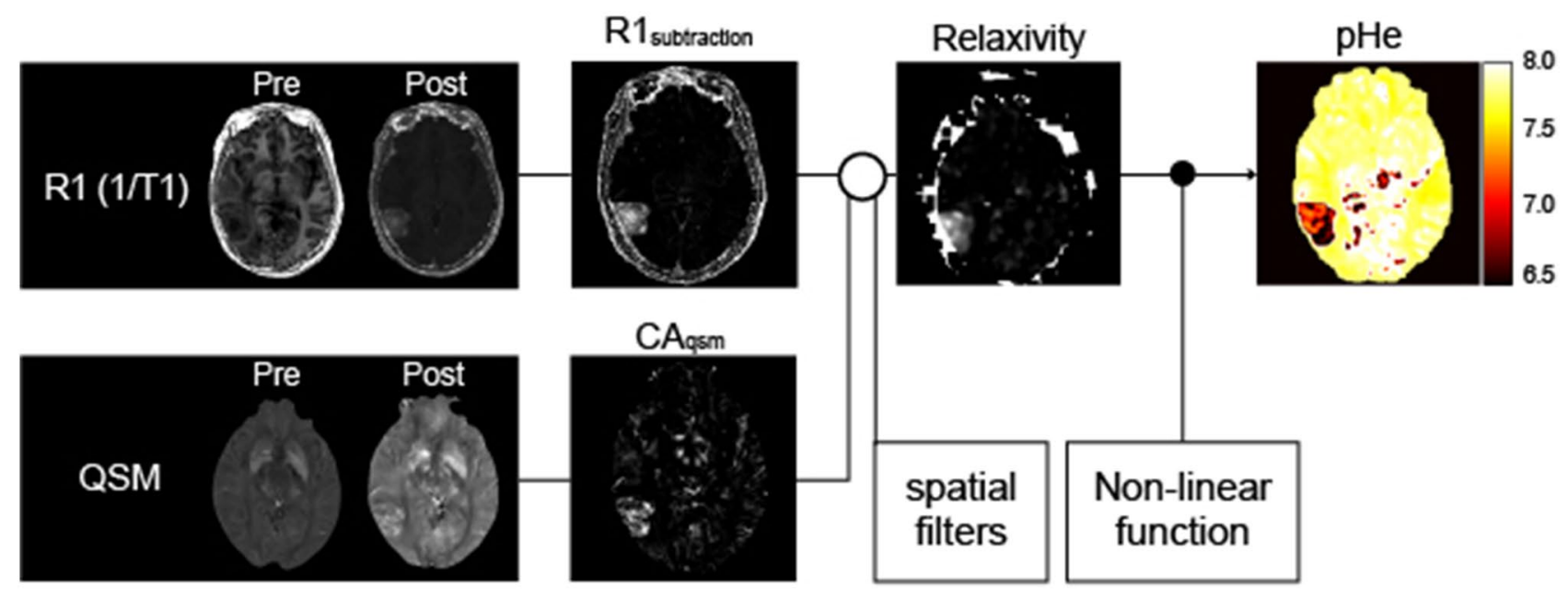

Figure 1. Scheme of the procedure for extracellular $\mathrm{pH}(\mathrm{pHe})$ calculation. Relaxivity $(\mathrm{r} 1)$ and susceptibility maps were calculated from both quantitative parameter mapping (QPM) datasets before and after contrast agent (CA) injection. The subtraction $\left(R_{1 \text { substraction }}\right)$ map and the CA concentration map $\left(\mathrm{CA}_{\mathrm{qsm}}\right)$ were then calculated from these R1 and susceptibility maps, respectively (see Eqs. 4 and 5). The relaxivity map was calculated by dividing the $R_{1 \text { substraction }}$ by $\mathrm{CA}_{\mathrm{qsm}}$ each voxel. The $\mathrm{pHe}$ was finally estimated by applying a $\mathrm{pH}$ calibration curve at body temperature $\left(37^{\circ} \mathrm{C}\right)$.

(eTEs) was used: $78.0 \mathrm{~ms}$. The other imaging parameters were as follows: field of view (FOV), $288 \mathrm{~mm}$; imaging matrices and reconstruction matrices, $288 \times 201.6 \mathrm{~mm}^{2}$; and slice thickness, $6 \mathrm{~mm}$.

Determination of the $\mathrm{pH}$-relaxivity curve. Here, we describe how the $\mathrm{pH}$-relaxivity calibration curve can be calculated from T1 maps. Non-linear least square fitting was first performed to yield longitudinal relaxation times $\mathrm{T} 1 \mathrm{using}$ the following equation ${ }^{13}$ :

$$
S_{T I}=a b s\left[M 0 \times\left(1-(1-k) \times \exp \left(\frac{-T I}{T 1}\right)\right)\right],
$$

where SI defines the MR signal, and the subscript TI refers to each variable obtained at TI. M0 and T1 define equilibrium magnetization and longitudinal relaxation times, respectively. The mean R1 value of each container was determined by drawing a region-of-interest (ROI), which was an equally sized square shape (64 pixels). After a linear regression analysis based on Eq. (1b) was performed, $r 1$ values were individually calculated at each $\mathrm{pH}$. In this study, the $\mathrm{pH}$-sensitive range was chosen as previously reported ${ }^{14}$ because it exhibits unique characteristics depending on the CA's chemical structure. The $\mathrm{pH}$ can then be calculated using least square fitting of Hill-modified Henderson-Hasselbalch equation:

$$
p H=p K a-\log _{10}\left[\frac{r 1-r 1_{\text {base }}}{r 1_{\text {acid }}-r 1}\right]^{n}
$$

where $\mathrm{r} 1$ defines relaxivity, and $\mathrm{pKa}, \mathrm{r}_{\text {base}}, \mathrm{r} 1_{\text {acid }}$, and $\mathrm{n}$ must be determined according to the phantom experiment.

Calculation of relaxivity values. An overview of data processing is provided in Fig. 1. There are several processing steps to estimate $\mathrm{r} 1$ before and after CA injection of the contrast agent. First, R1 and susceptibility maps were calculated from both QPM datasets before and after CA injection. The subtraction $\left(R_{1 \text { substraction }}\right)$ map and the CA concentration map $\left(\mathrm{CA}_{\mathrm{qsm}}\right)$ were then calculated as follows:

$$
\begin{gathered}
R 1_{\text {subtraction }}=R 1_{\text {post }}-R 1_{\text {pre }}, \\
C A_{q s m}=\frac{\chi_{p o s t}-\chi_{\text {pre }}}{\chi_{g d}} \times M o l_{G d},
\end{gathered}
$$

where $\chi_{\text {pre }}$ and $\chi_{\text {post }}$ define susceptibility values before and after CA injection, respectively, and $\chi_{G d}$ defines CA's susceptibility ${ }^{15}$. In addition, $\mathrm{Mol}_{G d}$ defines a CA's molar concentration. In this study, multiple dipole-inversion combination with k-space segmentation (MUDICK) and $326 \mathrm{ppm}$ were used for estimating $\chi_{\text {pre }}, \chi_{\text {post }}$ and $\chi_{G d}$, respectively ${ }^{16,17}$. The relaxivity $r_{1}$ was subsequently estimated with Eq. (1b) by measuring $R_{1 \text { subtraction }}$ and $\mathrm{CA}_{\mathrm{qsm}}$. Here, a linear regression analysis was performed to confirm linearity between the $\mathrm{CA}_{\mathrm{qsm}}$ and the $R_{1 \text { subtraction }}$ in the brain tumor. To remove division artifacts, a 3D median filter and Gaussian filter (standard deviation of 1) were applied to $r_{1}$. Finally, the pHe map was calculated based on the resulting $r_{1}$ map and the non-linear function derived from the phantom experiment. Of note, the $\mathrm{pHe}$ value can only be measured in contrast-enhanced areas 

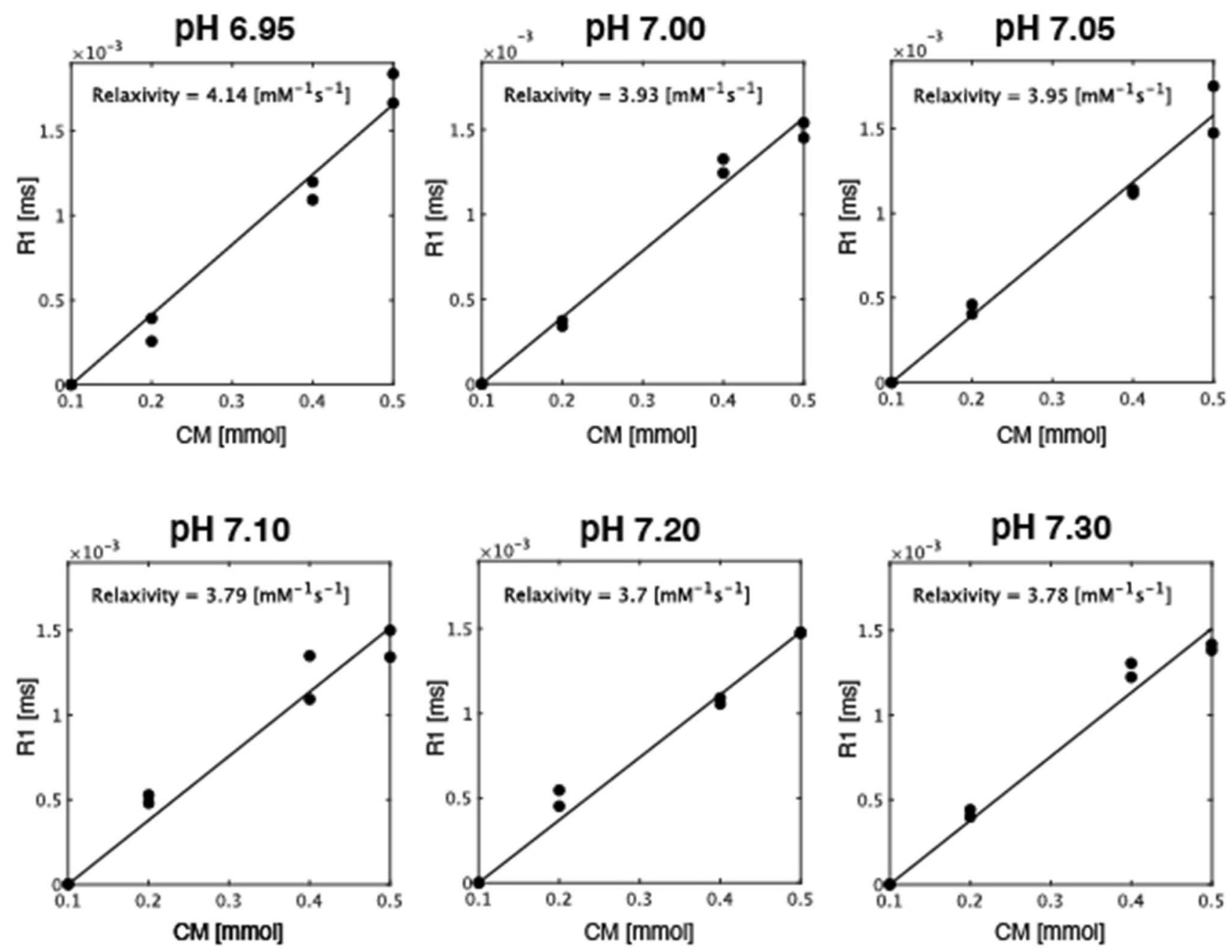

Figure 2. Determination of Gd-BTDO3A relaxivity at 3.0 T MRI. Relaxation rates (1/T1) were plotted against the concentration of four Gd-BTDO3A concentrations $(0.1,0.2,0.4$ and $0.5 \mathrm{mmol})$ at body temperature $\left(37^{\circ} \mathrm{C}\right)$, respectively. The relaxivity value was then determined from the slope of the linear regression of each $\mathrm{pH}$ solution.

caused by the disrupted blood-brain barrier. It is therefore impossible to compare the pHe value on the tumor region and that of normal tissue, such as normal appearing white matter.

Statistical analysis. After administering the contrast, ROIs were drawn on the R1 map and applied to the quantitative maps of $\mathrm{R} 1_{\text {subtraction }}, \mathrm{CA}_{\mathrm{gsm}}, \mathrm{rl}$ and $\mathrm{pHe}$. As the mean $\mathrm{pH}$ difference based on tumor malignancy would indicate the clinical potential of brain tumor $\mathrm{pHe}$, a one-way analysis of variance (ANOVA) test was used to observe whether changes in the mean $\mathrm{pHe}$ and $\mathrm{r} 1$ were dependent on malignancy. To estimate the $\mathrm{pH}$ value of the blood, ROIs were also drawn on the superior sagittal sinus using $\sim 10$ slices on the $\mathrm{R} 1_{\text {subtraction }}$ each patient and applied to the pHe map.

\section{Results}

Phantom experiment. Figure 2 shows the relaxivity determination of Gd-BTDO3A at 3.0 T MRI. Relaxation rates $(1 / \mathrm{T} 1)$ were plotted against the concentration of four Gd-BTDO3A examples at body temperature $\left(37^{\circ} \mathrm{C}\right)$. Figure 3 shows $\mathrm{r} 1$ depending on a $\mathrm{pH}$ range at room temperature and body temperature, respectively. The $\mathrm{pH}$ calibration curve of body temperature was defined as the $\mathrm{pH}$-sensitive ranges after confirming the relaxivity behavior from Fig. 2. In this study, a range of 6.95-7.3 was considered as a pH-sensitive range, and a non-linear regression analysis performed. The fitted result led to the following values: $\mathrm{kPa}=6.70, \mathrm{r} 1_{\text {base }}=3.50$, $\mathrm{r}_{\mathrm{acid}}=6.50$, and $\mathrm{n}=1.34$.

Brain tumor. The comparisons demonstrating a strong correlation between $\mathrm{CA}_{\mathrm{qsm}}$ and $\mathrm{R} 1$ subtraction maps of all tumor lesions are shown in Fig. $4\left(\mathrm{R}^{2} \geq 0.55\right)$. In this study, the relationship between $\mathrm{CA}_{\mathrm{qsm}}$ and $R_{1}$ subtraction was individually plotted because of the mean relaxivity value, defined as the slope of the resulting fit from the linear regression. The mean brain tumor $\mathrm{rl}$ changed with the anabolic metabolism in the extracellular space, 


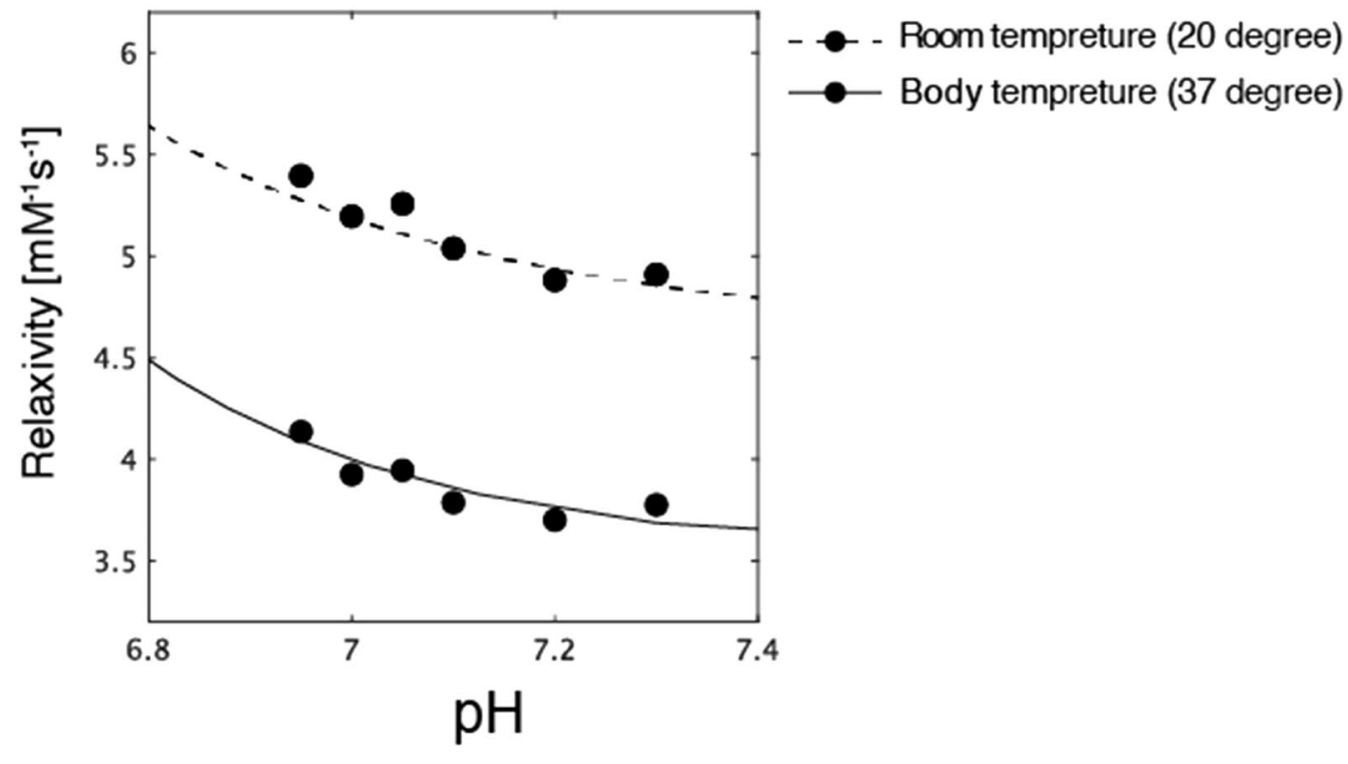

Figure 3. Determination of the pHe-relaxivity calibration curve for Gd-BTDO3A. These measurements were performed at room temperature $\left(20^{\circ} \mathrm{C}\right)$ and body temperature $\left(37^{\circ} \mathrm{C}\right)$ on a clinical $3.0 \mathrm{~T}$ MRI scanner.

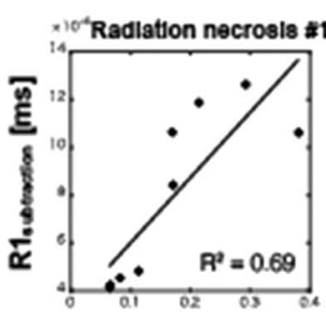

CMosen [mM]

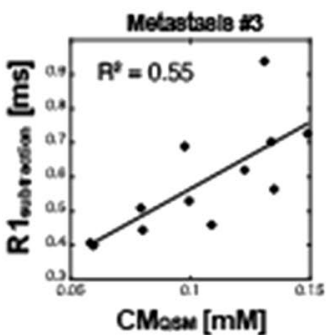

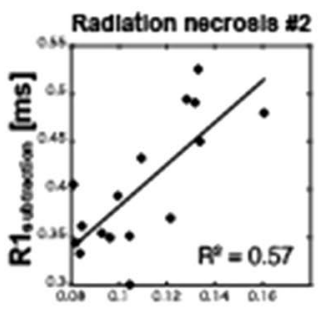

CMosu [mM]

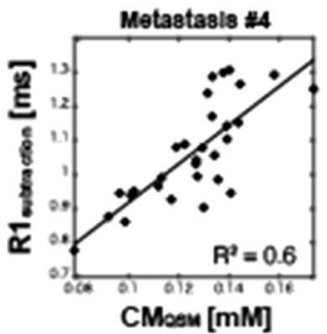

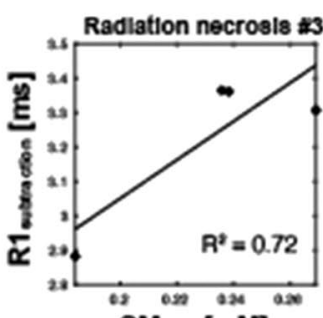

CMose $[\mathrm{mM}]$

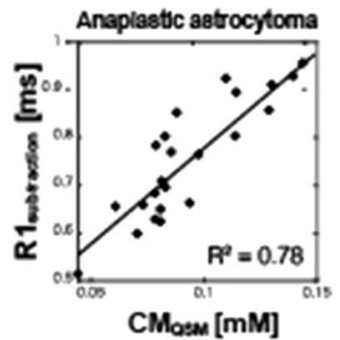

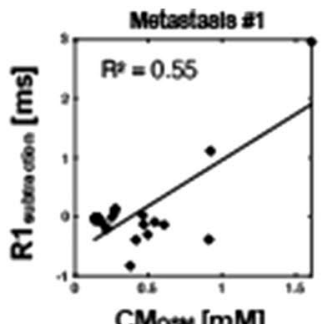

CMasu [mM]

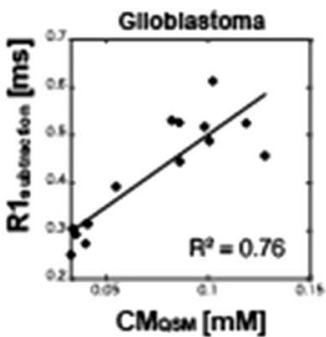

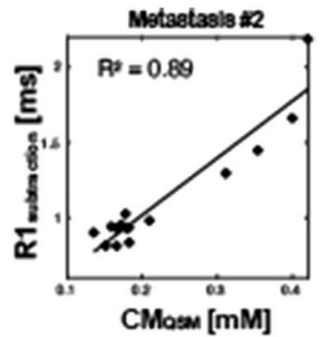

CMosu [mM]

Figure 4. Relationship between $R 1_{\text {subtraction }}$ and $C A_{\mathrm{qsm}}$ for each brain disease. Mean $R 1_{\text {subtraction }}$ values were plotted against mean $\mathrm{CA}_{\mathrm{qsm}}$ values on tumor lesions. The tumor lesions were defined by drawing region-ofinterest (ROI) on the R1 map after contrast agent injection.

indicating that CA's $\mathrm{r} 1$ can be independently measured by $\mathrm{R}_{1 \text { subtraction }}$ and $\mathrm{CA}_{\mathrm{qsm}}$. The mean values of $R_{1 \text { subtraction, }}$, $\mathrm{CA}_{\mathrm{qsm}}$, relaxivity, and $\mathrm{pHe}$ are shown in Fig. 5 . Table 2 also shows the mean values of $R_{1 \text { subtraction, }}, \mathrm{CA}_{\mathrm{qsm}}$, relaxivity, and $\mathrm{pHe}$ for each patient.

The primary brain tumor group showed significantly higher mean $\mathrm{r} 1$ values than other brain disease groups $(\mathrm{P}<0.001)$. Moreover, the mean $\mathrm{r} 1$ of the metastasis group was significantly increased compared with radiation necrosis $(\mathrm{P}<0.001)$. The mean $\mathrm{pHe}$ value showed a trend for tumor malignancy having a lower $\mathrm{pHe}$ value and primary brain tumor having a significantly lower $\mathrm{pHe}$ than other brain diseases $(\mathrm{P}<0.001)$. Moreover, the mean pHe value of the metastasis group was significantly decreased compared with that of the radiation necrosis, indicating that $\mathrm{pHe}$ can help evaluate therapeutic efficacy $(\mathrm{P}<0.001)$. Moreover, the synthetic $T_{1} \mathrm{w}$ post contrast administration derived from QPM and pHe maps is shown in Fig. 6. Mean pHe value of 7.49 \pm 0.019 was observed in the superior sagittal sinus. This value approximately matched the theoretical blood $\mathrm{pH}$ of 7.40. 

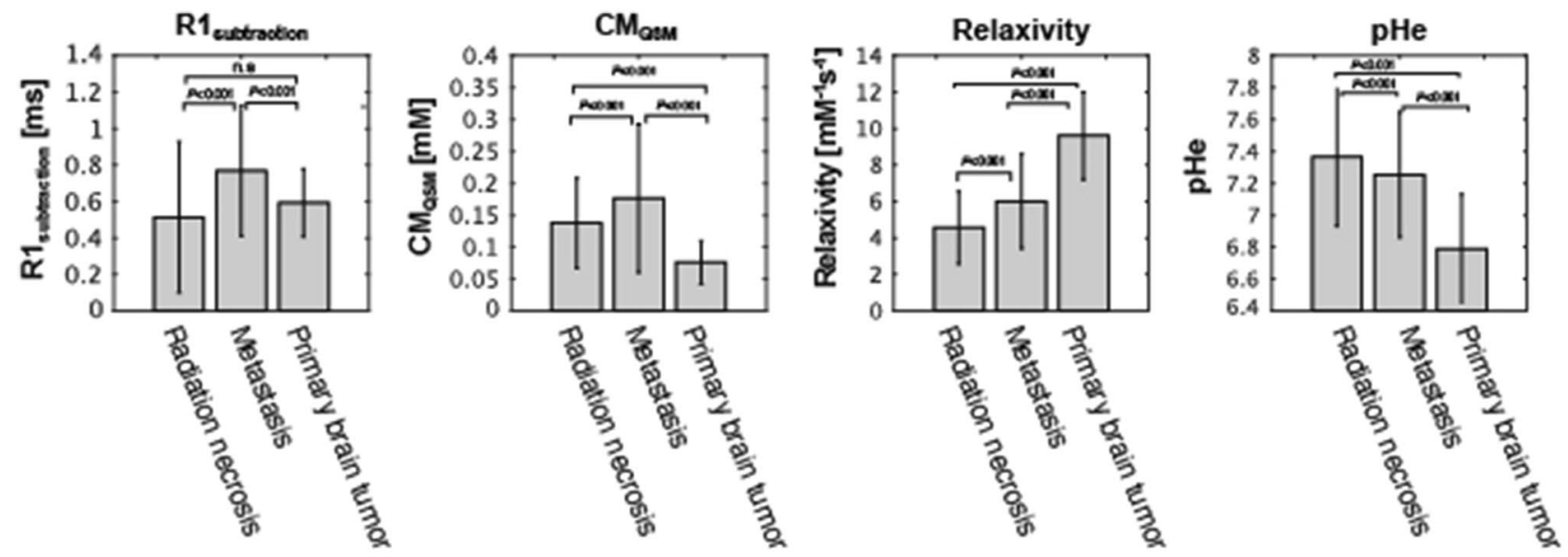

Figure 5. Mean $\mathrm{R} 1$ subtraction, $\mathrm{CA}_{\mathrm{qsm}}$, relaxivity, and $\mathrm{pHe}$ values among patient groups. The mean $\mathrm{pHe}$ value showed a trend for tumor malignancy having a lower $\mathrm{pHe}$ value and primary brain tumors having a significantly lower $\mathrm{pHe}$ than other brain diseases $(P<0.001)$.

\begin{tabular}{|l|l|l|l|l|l|}
\hline Age (years) & Sex & Clinical diagnosis & CA $(\mathbf{m m o l})$ & Relaxivity $\left(\mathbf{m M}^{-1} \mathbf{s}^{-1}\right)$ & pHe \\
\hline 79 & F & Radiation necrosis \#1 & $0.16 \pm 0.22$ & $0.01 \pm 0.00$ & $7.81 \pm 0.00$ \\
\hline 61 & F & Radiation necrosis \#2 & $0.13 \pm 0.12$ & $1.69 \pm 2.53$ & $7.34 \pm 0.90$ \\
\hline 72 & F & Radiation necrosis \#3 & $0.24 \pm 0.32$ & $4.61 \pm 3.39$ & $7.12 \pm 0.63$ \\
\hline 68 & M & Metastasis \#1 & $0.23 \pm 0.32$ & $11.32 \pm 6.64$ & $7.75 \pm 0.64$ \\
\hline 72 & F & Metastasis \#2 & $0.11 \pm 0.13$ & $5.23 \pm 2.09$ & $6.97 \pm 0.71$ \\
\hline 74 & M & Metastasis \#3 & $0.23 \pm 0.19$ & $5.91 \pm 2.41$ & $6.79 \pm 0.67$ \\
\hline 47 & F & Metastasis \#4 & $0.13 \pm 0.12$ & $9.4 \pm 4.50$ & $6.95 \pm 0.66$ \\
\hline 49 & F & Anaplastic astrocytoma & $0.08 \pm 0.07$ & $9.54 \pm 4.74$ & $6.79 \pm 0.81$ \\
\hline 76 & F & Glioblastoma & $0.05 \pm 0.05$ & $9.82 \pm 5.01$ & $6.79 \pm 0.99$ \\
\hline
\end{tabular}

Table 2. Mean CA, relaxivity, and pHe values each of the patients.

\section{Discussion}

r1-based CAs have an important potential application in pHe measurement. In the early days, the r1 values were reported to show an increasing/decreasing behavior depending on the $\mathrm{pH}^{14}$. This behavior is based on protic exchange and second hydration sphere dynamics that can provide the relaxivity change in acidic environments; therefore, the acidic environment plays a role in modulating relaxation changes ${ }^{13,18,19}$. We first performed a phantom study to determine the $\mathrm{pH}$ sensitivity of a conventional CA, Gd-BTDO3A. In this experiment, a range of 6.95-7.3 was considered as the $\mathrm{pH}$-sensitive range for the calibration curve, resulting in four parameters: $\mathrm{kPa}=6.70, \mathrm{r} 1$ base $=3.50, \mathrm{r} 1 \mathrm{acid}=6.50$ and $\mathrm{n}=1.34$. The relaxivity value depends on temperature because the relaxation time of $\mathrm{T} 1$ changes linearly with the temperature ${ }^{20}$. In this study, the relationship between relaxivity and $\mathrm{pH}$ at room temperature and body temperature (Fig. 3) supports the notion of temperature dependence of relaxivity.

In our approach to separately quantify CA concentration and r1, we used QPM because it can simultaneously measure the quantitative maps of both relaxation and susceptibility, leading to a clinically feasible acquisition time ( $20 \mathrm{~min})$. We then measured QPM in patients before and after administering Gd-BTDO3A and separately quantified the CA concentration and $\mathrm{r} 1$ from $R_{1 \text { subtraction }}$ and $\mathrm{CA}_{\mathrm{qsm}}$ maps. Both maps had a high correlation with each brain disease (see Fig. 4 ). This means that $R_{1 \text { subtraction }}$ and $\mathrm{CA}_{\mathrm{qsm}}$ maps can separately detect the Gd3+ relaxation change in tumor lesions. Thus, QPM has an important practical advantage for detecting Gd3+ behavior on clinical scan time. An additional advantage of QPM is that standard T1w, T2w, T2* w, fluid-attenuated inversion recovery (FLAIR) images can be simultaneously obtained both before and after CA injection, as shown in Fig. 7, which displays actual post-processed synthetic MRI data of a patient with metastasis \#4. According to the present results, using QPM to determine T1w, T2w, T2* ${ }^{\star}$, FLAIR, and pHe estimates would lead to more accurate characterization and identification of brain lesions.

A pHe map was then obtained by applying the result of the phantom experiment to the $\mathrm{r} 1 \mathrm{map}$, showing that the mean pHe value decreased in the tumor grade group, as shown in Fig. 5. This observation is consistent with that of earlier studies showing that the average pHe reaches approximately 6.8-7.2 in solid tumors because of the Warburg effect ${ }^{21-23}$. Additionally, the blood $\mathrm{pHe}$ was measured on the superior sagittal sinus to investigate whether the blood $\mathrm{pH}$ can be correctly estimated. As a result, the mean value of $7.49 \pm 0.019$ was observed. This 


\section{Radiation}

necrosis

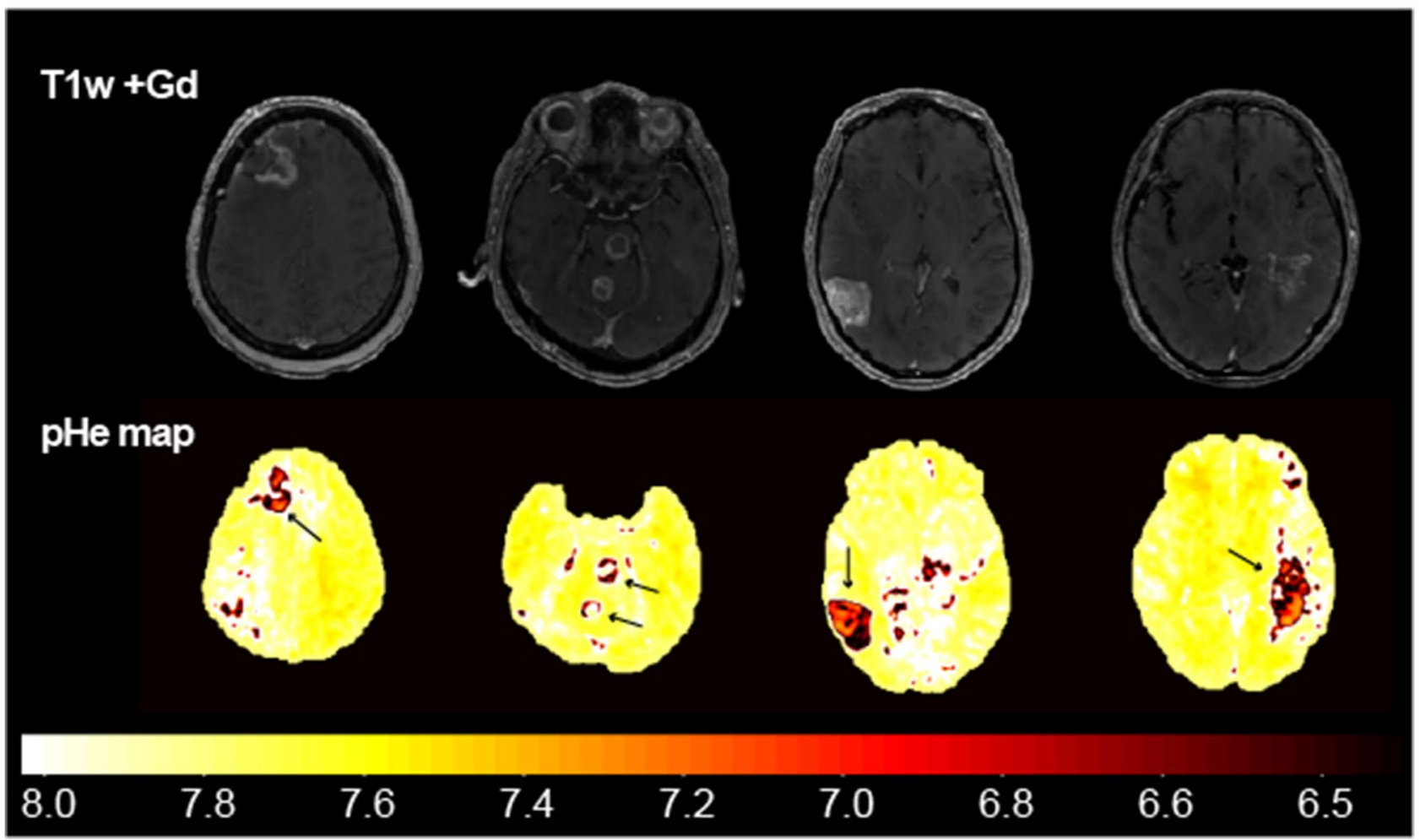

Figure 6. Contrast-enhanced synthetic T1w image-derived QPM and pHe maps. The synthetic T1w and pHe maps indicate contrast-enhanced lesions and $\mathrm{pHe}$ changes with a color scale bar [pH 6.5-8.0].

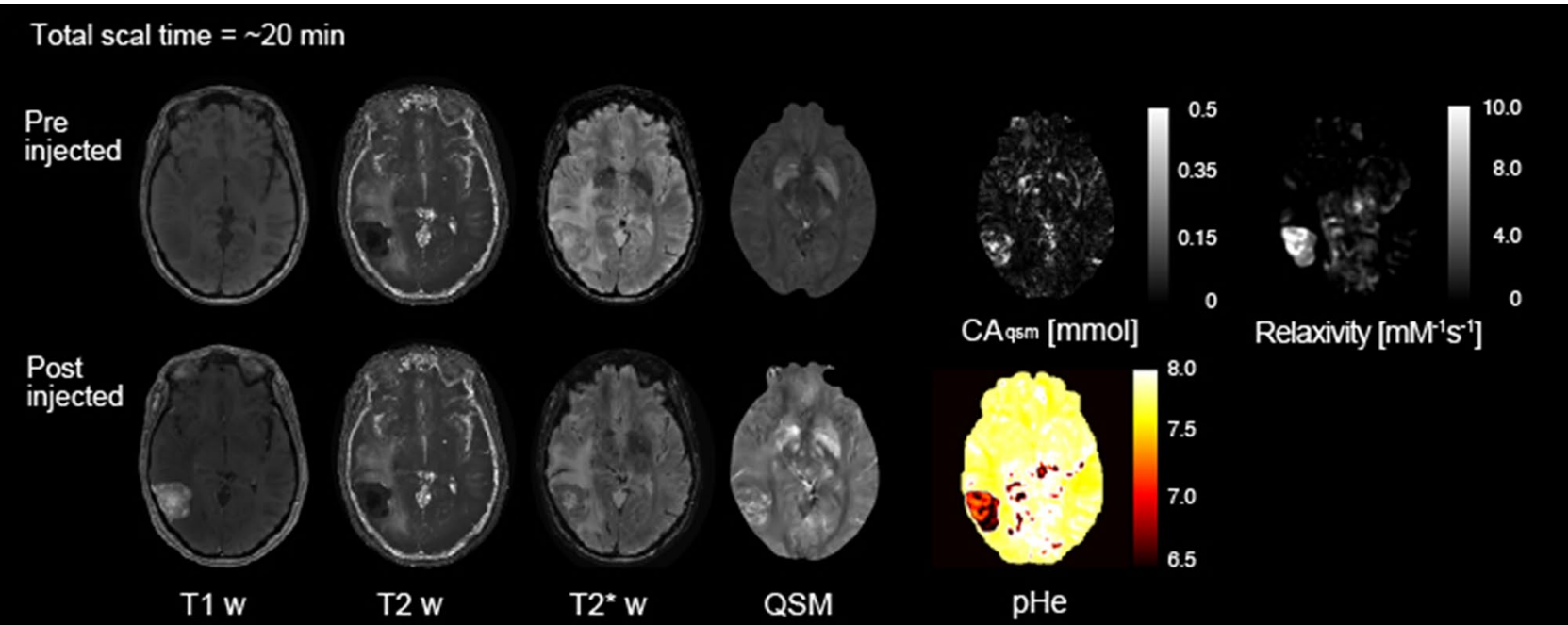

Figure 7. Brain disease evaluation using QPM before and after contrast agent injection. Simultaneous 3D mapping of $\mathrm{PD}, \mathrm{T} 1, \mathrm{~T} 2{ }^{\star}$ and susceptibility map can obtain contrast images. Moreover, our method can estimate CA concentration, $\mathrm{r} 1$ and $\mathrm{pHe}$ mapping by using $\mathrm{CA}$.

value approximately matched the theoretical blood $\mathrm{pH}$ of 7.40 , our approach can approximately quantify $\mathrm{pHe}$, if relaxivity values are not high.

Our study proposes a new method using QPM to evaluate $r 1$ and CA concentration in contrast-enhanced lesions, showing the possibility of evaluating tumors' pathophysiological changes. Furthermore, r1 values may be used as pHe markers. These indices may be useful for brain tumor management.

A major limitation of the conventional CA based pHe measurement is that sensitivity to CA is limited in a narrow $\mathrm{pH}$ range in clinical measurements. In this study, a pHe-relaxivity calibration curve was obtained with 
Radiation necrosis \#1
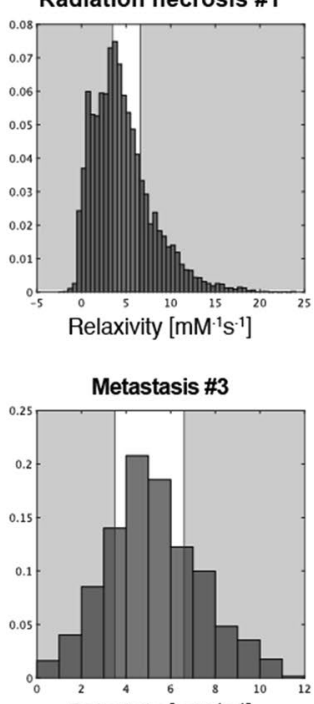

Relaxivity $\left[\mathrm{mM}^{-1} \mathrm{~s}^{-1}\right]$
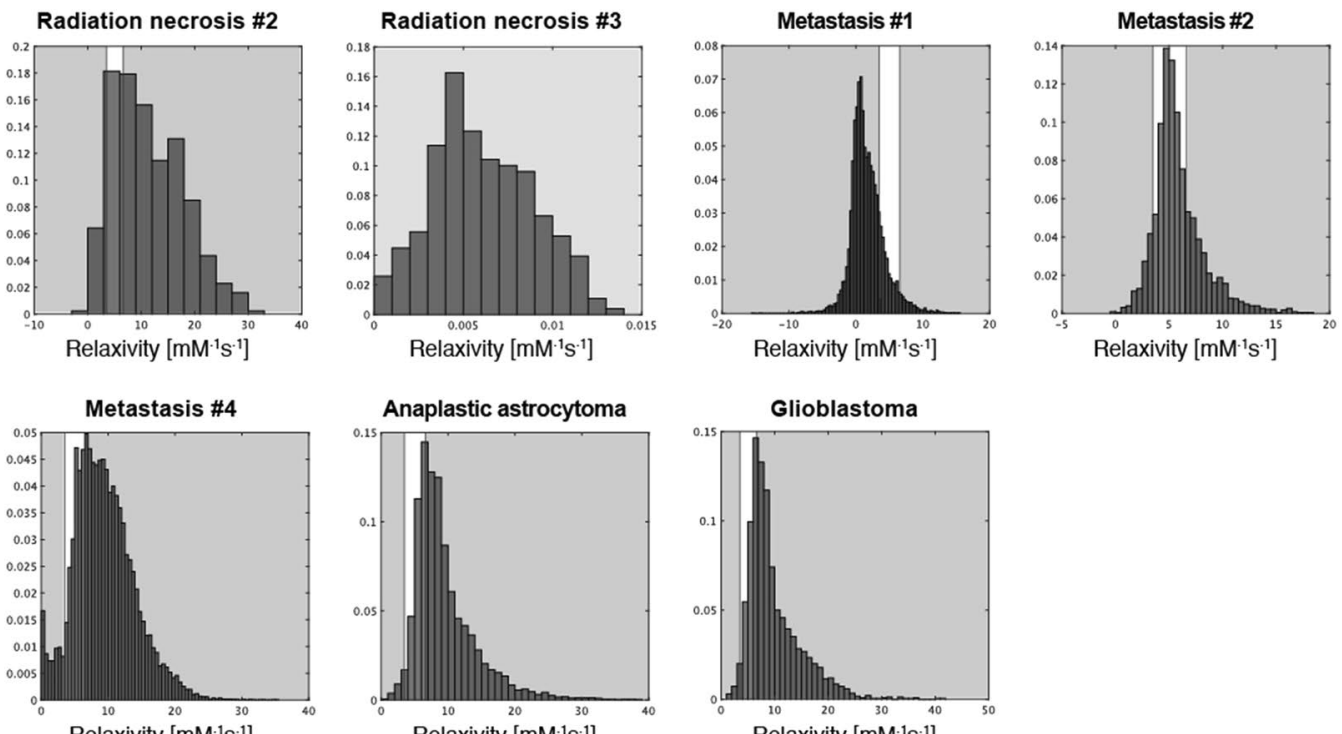

Relaxivity $\left[\mathrm{mM}^{\cdot 1} \mathrm{~s}^{-1}\right]$

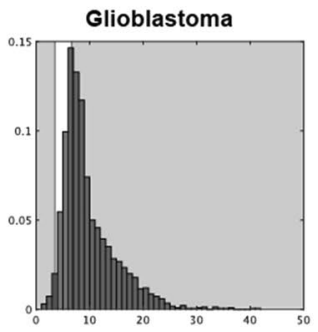

Relaxivity $\left[\mathrm{mM}^{\cdot 1} \mathrm{~s}^{\cdot 1}\right]$

Figure 8. Histogram of relaxivity in the tumor lesion for each patient. White and gray areas indicate $\mathrm{pH}$ sensitive and insensitive ranges against the relaxivity distribution, respectively.

four parameters: $\mathrm{kPa}=6.70, \mathrm{rl}_{\text {base }}=3.50, \mathrm{r}_{\text {acid }}=6.50$ and $\mathrm{n}=1.34$, indicating a sensitivity range from 3.50 to 6.50 . Figure 8 shows a histogram of the relaxivity in the tumor lesion for each patient. As a result, a narrow sensitive range (white area in Fig. 8) was observed against the relaxivity distribution, which is in agreement with the peak of the relaxivity distribution except for radiation necrosis \#3 and Metastasis \#1. Additionally, we believe our results to be acceptable because the mean pHe value significantly decreased in subjects with primary tumors compared to that in those with radiation necrosis. In future work, a newly developed $\mathrm{pH}$-sensitive contrast agent may be required to increase the range and accuracy of $\mathrm{pH}$ sensitivity. Although TmDOTP5 has been studied as a $\mathrm{pH}$ sensitive CA, it is not approved for clinical use ${ }^{24-26}$. Another major limitation is that despite showing the $\mathrm{pH}$-relaxivity curve, the accuracy of $\mathrm{pH}$ obtained using our methodology has not been validated in detail. Further in vitro studies using QPM are needed to determine the $\mathrm{pH}$ accuracy. QPM was optimized to achieve adequate relaxation time and susceptibility mapping of brain tissue. Therefore, at present, simultaneous imaging of both $\mathrm{T} 1$ and susceptibility mapping are difficult in a phantom experiment.

Our pHe measurement is specifically designed for the acidic extracellular space of solid tumors. However, our method is not restricted to brain tissue computation and can be adapted to other tissues as data acquisition is based on 3D spoiled gradient echo pulse sequences. Therefore, our methods could assist in the future application of pHe mapping to other tissues.

In conclusion, this study demonstrated that QPM can separately quantify $\mathrm{r} 1$ and CA concentration in brain tumors and that $\mathrm{pHe}$ mapping of brain tumors could serve as a biomarker for tumor characterization. In particular, our method has clinical potential for assessing the treatment effects.

\section{Data availability}

The authors confirm that the data supporting the findings of this study are available within the article.

Received: 16 July 2021; Accepted: 18 January 2022

Published online: 09 February 2022

\section{References}

1. Reichenbach, J. et al. $1 \mathrm{H} \mathrm{T1}$ and T2 measurements of the MR imaging contrast agents Gd-DTPA and Gd-DTPA BMA at $1.5 \mathrm{~T}$. Eur. Radiol. 7, 264-274 (1997).

2. Mikawa, M., Miwa, N., Bräutigam, M., Akaike, T. \& Maruyama, A. Gd3+-loaded polyion complex for pH depiction with magnetic resonance imaging. J. Biomed. Mater. Res. 49, 390-395 (2000).

3. Gerweck, L. The $\mathrm{pH}$ difference between tumor and normal tissue offers a tumor specific target for the treatment of cancer. Drug Res. Updat. 3, 49-50 (2000).

4. Lin, S. \& Brown, J. MR contrast agents: Physical and pharmacologic basics. J. Magn. Reson. Imaging. 25, 884-899 (2007).

5. Vibhute, S. et al. Synthesis and characterization of $\mathrm{pH}$-sensitive, biotinylated MRI contrast agents and their conjugates with avidin. Org. Biomol. Chem. 11, 1294-1305 (2013).

6. Verwilst, P., Park, S., Yoon, B. \& Kim, J. Recent advances in Gd-chelate based bimodal optical/MRI contrast agents. Chem. Soc. Rev. 44, 1791-1806 (2015).

7. Weiskopf, N. et al. Quantitative multi-parameter mapping of R1, PD*, MT, and R2* at 3T: A multi-center validation. Front. Neurosci. 7, 95 (2013).

8. Garcia-Martin, M. et al. High resolution pHe imaging of rat glioma using pH-dependent relaxivity. Magn. Reson. Med. 55, 309-315 (2006).

9. Martinez, G. et al. Imaging the extracellular $\mathrm{pH}$ of tumors by MRI after injection of a single cocktail of T1 and T2contrast agents. NMR Biomed. 24, 1380-1391 (2011). 
10. Laus, S., Sour, A., Ruloff, R., Tóth, É. \& Merbach, A. Rotational dynamics account for pH-dependent relaxivities of PAMAM dendrimeric, Gd-based potential MRI contrast agents. Chemistry 11, 3064-3076 (2005).

11. Aime, S., Fedeli, F., Sanino, A. \& Terreno, E. A R2/R1Ratiometric procedure for a concentration-independent, pH-responsive, Gd(III)-based MRI agent. J. Am. Chem. Soc. 128, 11326-11327 (2006).

12. Stack, J., Redmond, O., Codd, M., Dervan, P. \& Ennis, J. Breast disease: Tissue characterization with Gd-DTPA enhancement profiles. Radiology 174, 491-494 (1990).

13. Rohrer, M., Bauer, H., Mintorovitch, J., Requardt, M. \& Weinmann, H. Comparison of magnetic properties of MRI contrast media solutions at different magnetic field strengths. Invest. Radiol. 40, 715-724 (2005).

14. Zhang, S., Wu, K. \& Sherry, A. A novel pH-sensitive MRI contrast agent. Angew. Chem. Int. Ed. Engl. 38, 3192-3194 (1999).

15. Lind, E., Knutsson, L., Kämpe, R., Ståhlberg, F. \& Wirestam, R. Assessment of MRI contrast agent concentration by quantitative susceptibility mapping (QSM): Application to estimation of cerebral blood volume during steady state. MAGMA 30, 555-566 (2017).

16. Sato, R. et al. Quantitative susceptibility mapping using the multiple dipole-inversion combination with k-space segmentation method. Magn. Reson. Med. Sci. 16, 340-350 (2017).

17. de Rochefort, L., Brown, R., Prince, M. \& Wang, Y. Quantitative MR susceptibility mapping using piece-wise constant regularized inversion of the magnetic field. Magn. Reson. Med. 60, 1003-1009 (2008).

18. Reuben, J. Gadolinium(III) as a paramagnetic probe for proton relaxation studies of biological macromolecules. Binding to bovine serum albumin. Biochemistry 10, 2834-2838 (1971).

19. Stanisz, G. \& Henkelman, R. Gd-DTPA relaxivity depends on macromolecular content. Magn. Reson. Med. 44, 665-667 (2000).

20. Lu, H., Clingman, C., Golay, X. \& van Zijl, P. Determining the longitudinal relaxation time (T1) of blood at 3.0 Tesla. Magn. Reson. Med. 52, 679-682 (2004).

21. Helmlinger, G., Yuan, F., Dellian, M. \& Jain, R. Interstitial $\mathrm{pH}$ and $\mathrm{pO}_{2}$ gradients in solid tumors in vivo: High-resolution measurements reveal a lack of correlation. Nat. Med. 3, 177-182 (1997).

22. Warburg, O., Wind, F. \& Negelein, E. The metabolism of tumors in the body. J. Gen. Physiol. 8, 519-530 (1927).

23. Gillies, R. \& Gatenby, R. Hypoxia and adaptive landscapes in the evolution of carcinogenesis. Cancer Metastasis Rev. 26, 311-317 (2007).

24. Sun, Y. et al. Simultaneous measurements of temperature and $\mathrm{pH}$ in vivo using NMR in conjunction with TmDOTP5?. NMR Biomed. 13, 460-466 (2000).

25. Coman, D. et al. Imaging the intratumoral-peritumoral extracellular pH gradient of gliomas. NMR Biomed. 29, 309-319 (2016).

26. Rao, J. et al. Temozolomide arrests glioma growth and normalizes intratumoral extracellular pH. Sci. Rep. 7(1), 1-6 (2017).

\section{Acknowledgements}

This work was supported by JSPS KAKENHI Grant number JP20K16759.

\section{Author contributions}

Author contributions here are given by referring Contributor Roles Taxonomy (CRediT). Y.M.: conceptualization, data curation, formal analysis, funding acquisition, investigation, software, visualization, writing-original draft. M.H.: conceptualization, funding acquisition, investigation, supervision, project administration, writing-review and editing. Y.K.: supervision, writing-review and editing. Y.T., M.O., B.Y.: software, resources.

\section{Funding}

This study was funded by FUJIFILM Healthcare Corporation.

\section{Competing interests}

Y.M.: This work was supported by JSPS KAKENHI Grant Number JP20K16759. M.H: He has been funded by FUJIFILM Healthcare Corporation. Y.K.: He declares no potential conflict of interest. Y.T., M.O., B.Y.: Employees of the FUJIFILM Healthcare Corporation, Japan and the authors have conflict of interest to disclose with respect to this study.

\section{Additional information}

Correspondence and requests for materials should be addressed to Y.M.

Reprints and permissions information is available at www.nature.com/reprints.

Publisher's note Springer Nature remains neutral with regard to jurisdictional claims in published maps and institutional affiliations.

(c) (i) Open Access This article is licensed under a Creative Commons Attribution 4.0 International License, which permits use, sharing, adaptation, distribution and reproduction in any medium or format, as long as you give appropriate credit to the original author(s) and the source, provide a link to the Creative Commons licence, and indicate if changes were made. The images or other third party material in this article are included in the article's Creative Commons licence, unless indicated otherwise in a credit line to the material. If material is not included in the article's Creative Commons licence and your intended use is not permitted by statutory regulation or exceeds the permitted use, you will need to obtain permission directly from the copyright holder. To view a copy of this licence, visit http://creativecommons.org/licenses/by/4.0/.

(c) The Author(s) 2022 